\title{
Invest in Bone Health: Prevent Osteoporosis
}

\author{
Nizar Abdul Majeedkutty* \\ Department of Physiotherapy, Malaysia
}

*Corresponding author: Nizar Abdul Majeedkutty, Senior Lecturer \& Head of Department, Department of Physiotherapy, Malaysia

\begin{abstract}
Osteoporosis is a largely preventable disease that is characterized by bones that are porous and have a low density. Osteoporosis is associated with an increased risk of fractures. This condition can often be reduced, eliminated, or prevented by following healthy lifestyle guidelines. Public health leaders and advocates, government and non-governmental agencies, communities, health-care professionals are responsible for preventing osteoporosis. Nutritional deficiency, hormonal disorders, lack of exercise, immobilization and smoking can lead to poor development of bone and accelerate the loss of bone mass. Awareness and understanding are the keys to reducing the overwhelming incidence of osteoporosis. Great progress has been made in reaching out to the public through health campaigns, research, and publications; however, as the statistics show, the fight is not over.
\end{abstract}

\section{Introduction}

Osteoporosis poses a significant public health issue, causing significant morbidity and mortality. Osteoporosis is a disease in which the density and quality of bone are reduced. This disease is characterized by bone fragility and an increased susceptibility to fractures, especially of the spine, hip and wrist, although any bone can be affected. The likelihood of these fractures increases with age in both women and men. The loss of bone occurs silently and progressively. Often there are no symptoms until the first fracture occurs. Around the world, 1 in 3 women and 1 in 5 men are at risk of an osteoporotic fracture. In fact, an osteoporotic fracture is estimated to occur every 3 seconds [1]. In adults, the daily removal of small amounts of bone mineral, a process called resorption, must be balanced by an equal deposition of new mineral if bone strength is to be preserved. When this balance tips toward excessive resorption, bones weaken (osteopenia) and over time can become brittle and prone to fracture (osteoporosis). There is evidence that fracture risk is increased in people on prolonged use of nonsteroidal anti-inflammatory drugs and glucocorticoid therapy.

\section{Who is at risk?}

Awareness of risk factors can help to take steps to reduce bone mineral loss. Fixed risk factors like disorders and medications can weaken bone and affect balance [2]. Fixed risk factors include increasing age, female gender, family history of osteoporosis, ethnicity, menopause, hysterectomy, rheumatoid arthritis, hypogonadism in men etc. Most modifiable risk factors like alcohol, smoking, low body mass index, poor nutrition, vitamin D deficiency, eating disorders, physical inactivity also can directly impact bone biology and result in a decrease in bone mineral density (BMD).

\section{Preventing osteoporosis}

It is now known that osteoporosis is a preventable and treatable disease and not a normal part of ageing [3]. Although genetic factors play a significant role in determining whether an individual is at heightened risk of osteoporosis, lifestyle factors such as diet and physical activity also influence bone development in youth and the rate of bone loss later in life. After mid-20s, bone thinning is a natural process and cannot be completely stopped. The thicker the bones, the less likely they are to become thin enough to break. Young women in particular need to be aware of their osteoporosis risk and take steps to slow its progress and prevent fractures. Optimal bone growth and development in youth is vital in the prevention of osteoporosis as it is an important determinant of the risk of osteoporotic fracture during later life. It is estimated a $10 \%$ increase of peak bone mass in children reduces the risk of an osteoporotic fracture during adult life by $50 \%$ [4]. Once peak bone mass has been reached, it is maintained by a process called remodeling. This is a continuous process in which old bone is removed and new bone 
is created. The renewal of bone is responsible for bone strength throughout life. Factors that cause a higher rate of bone remodeling will ultimately lead to a more rapid loss of bone mass and high risk of fractures. Adequate calcium levels are crucial for bone health and muscle performance, which are closely associated with balance and fall risk [5]. Vitamin D plays a major role to maintain serum calcium levels through enhancement of small-intestine absorption [6]. Sunlight exposure, fortified foods, egg yolks, saltwater fish, liver, are rich sources of Vitamin D. Due to increasingly indoor lifestyles, young people often don't get enough vitamin D. Parents should encourage children spend more time participating in sports and outdoor physical activity and less screen time in front of computers or televisions to maintain a healthy level of this key vitamin.

\section{How is osteoporosis diagnosed?}

Though traditional X-rays cannot measure bone density, they can identify spine fractures. Dual energy X-ray absorptiometry (DEXA) is the best technique for diagnosis and monitoring therapy. It is a low radiation X-ray used to measure spine and hip bone density and can also measure bone density of the whole skeleton. Other methods for diagnosing osteoporosis like Bone Turnover Markers (BTM) and radiological assessments have been used extensively in clinical trials and epidemiological studies.

\section{Medical Management}

Osteoporosis is potentially the most serious of menopause symptoms. The maximum bone loss occurs in first two years of menopause. Estrogen hormone therapy after menopause (previously referred to as hormone replacement therapy or HRT) has been shown to prevent bone loss, increase bone density, and prevent bone fractures. Estrogen is available orally, as a skin patch and in combination with progesterone as pills and patches. Progesterone given routinely along with estrogen helps prevent uterine cancer that might result from estrogen use alone [7]. It was thought that hormone therapy could ward off heart disease, osteoporosis, and cancer, while improving women's quality of life. The findings emerged from clinical trials showed that long-term use of hormone therapy poses serious risks and may increase the risk of heart attack and stroke. Bisphosphonates are the most commonly prescribed medications to treat osteoporosis [8]. These drugs slow bone loss by causing certain cells involved in bone breakdown to undergo programmed cell death.

\section{Regular exercise is essential}

Regular exercise helps decreasing the risk of falls, probably because balance is improved, and muscle strength is increased. Build up slowly and aim to gradually increase the repetitions of each exercise over time. It is important to avoid exercises that can injure already weakened bones. In patients over 40 and those with heart disease, obesity, diabetes mellitus, and high blood pressure, exercise should be prescribed and monitored by physicians. Extreme levels of exercise like lifting heavy weights and forward bending may not be healthy for the bones. Exercises to correct postural deformities like Dowager's hump should be incorporated in therapy.

\section{Weight-bearing exercises}

Several studies demonstrate the health benefits of exercise, including reduced risk of falls and fractures. Weight-bearing and muscle-strengthening exercises are ideal for osteoporosis prevention because it improves agility, posture, balance, and strength to prevent falls [5]. A statistically significant effect of exercise on BMD was reported by a systematic review investigating whether exercise could prevent bone loss and fractures in postmenopausal women [9]. It was found that high-force exercise involving lower limbs was the most effective exercise for femur neck BMD. Brisk walking is highly recommended, and patients can adapt their speed to the current fitness level. Sit-to-stand, Mini-squats, Calf raises, Wall press-up, Push-ups are good examples of exercises that patients can perform at home. Low-impact weight-bearing exercises like aerobics, using stair-step machines, fast walking on a treadmill are advised if high force exercises are contraindicated.

\section{Muscle-strengthening exercises}

Numerous studies have shown that strength training can play a role in slowing bone loss, and several show it can even build bone $[10,11]$. This is tremendously useful to help offset age-related declines in bone mass. Activities that put stress on bones can nudge bone-forming cells into action. Body weight exercises like squats, sit to stand; rising up on your toes can be performed at home. Lifting weights, using elastic exercise bands and using weight machines use muscle strength, where the action of the tendons pulling on the bones boosts bone strength. High-impact weight-bearing exercises help build bones and keep them strong. Cycling and swimming do not cause positive effects on BMD; thus, these are not the most suitable exercises for prevention and treatment of osteoporosis [12]. Swimming is neither a weight-bearing exercise nor a strengthtraining exercise. This means that if you are trying to prevent or fight osteoporosis, swimming should not be the only workout that you do.

\section{Diet is Important}

Many of the nutrients and food components we consume may influence bone by various mechanisms, including alteration of bone structure, the rate of bone metabolism, the endocrine and/ or paracrine system, and homeostasis of calcium and possibly of other bone-active mineral elements [13]. The most important nutrients for people with osteoporosis are calcium and vitamin D. Vitamin D helps body absorb calcium. Postmenopausal and senile osteoporosis can be prevented by adequate calcium intake during growth. Due to the accelerated muscular, skeletal and endocrine development, reduced intake of calcium and vitamin D during periods of growth can have a negative influence on bone development [14]. Bone mineral deposition during pubertal growth appears to depend on dietary absorption of calcium, and on 
reducing its excretion, and this is dependent on adequate Vitamin D status. A well-balanced diet with plenty of milk, fish, fruits and vegetables, cheese, and yogurt is important for bone health. The presence of lactose, caseinate and citrate in milk and dairy products helps better calcium absorption in relation to other dietary calcium sources. Vegetables with dark green leaves are also sources of calcium, but the calcium they contain has low bioavailability [15]. Osteoporosis experts recommend 800 to 1,200IU of vitamin D per day.

\section{Preventing falls}

Fall prevention helps prevent osteoporosis-related morbidity. Interventions include vision and hearing correction, removing trip or fall hazards, evaluating suspected neurologic problems, avoiding medications that cause imbalance, and advising hip pad protectors for those with significant risk. A person with osteoporosis is especially at risk of breaking bones from falling because the bones are so much weaker than normal healthy bones. Certain steps that can make a house safer for someone with osteoporosis is shown in Table 1.

Table 1: Steps to prevent falls.

\begin{tabular}{|c|c|}
\hline a. & Wear appropriate, flat shoes \\
\hline b. & $\begin{array}{l}\text { Wear glasses with the correct prescription and have this } \\
\text { checked regularly }\end{array}$ \\
\hline c. & Avoid having loose rugs and carpets or trailing electrical flexes \\
\hline d. & Make sure the home is well lit \\
\hline e. & $\begin{array}{c}\text { Be extra careful when taking medicines, especially sleeping } \\
\text { pills and sedatives that may cause drowsiness, making a person } \\
\text { more likely to trip or stumble }\end{array}$ \\
\hline f. & Keep the light in washroom switched on during night \\
\hline
\end{tabular}

The most effective treatment is provided by multidisciplinary approach involving physical therapist, psychologist, and nutritionist for consultation. Clinicians should consider risk-assessment to estimate absolute fracture risk and appropriate pharmacologic agents to prevent osteoporosis are to be prescribed [16]

\section{Life style factors}

Maintaining healthy habits can also reduce the risk or severity of osteoporosis. Smoking is an independent risk factor for osteoporosis as it reduces the amount of estrogen the body produces, and alcohol hinders calcium absorption [17]. It is also critical to maintain a healthy body weight. Nutrition is a modifiable pathogenic factor for osteoporosis. A holistic approach to bone health combining nutrition, physical activity and fall prevention is the key. Impactful public health education initiatives can create awareness about osteoporosis. Social media like Facebook groups and Twitter that provide a number of tools and resources as well as a forum for people to discuss osteoporosis shall be considered. Community programs with non-profit organizations reinforced at the local level can extend the program's reach and messages.

\section{Conclusion}

Studies should be conducted to quantify the status of bone health of at-risk population, their level of awareness and steps to improve bone health. This small investment will pay rich dividends in the long run by preventing the occurrence of fractures and improving the bone health and quality of life. Health workers must work together to improve health literacy and prevention awareness to ensure that people understand the principles of bone health.

\section{References}

1. International Osteoporosis Foundation. Retrieved on 30 Nov 2018.

2. Naz SGM, Ozgoli G, Aghdashi MA, Salmani F (2015) Prevalence and risk factors of osteoporosis in women referring to the bone densitometry academic center in Urmia, Iran. Glob J Health Sci 8(7): 135-145.

3. Osteoporosis prevention, diagnosis, and therapy (2000) NIH Consens Statement 17(1): 1-36.

4. Lu J, Shin Y, Yen MS, Sun SS (2014) Peak bone mass and patterns of change in total bone mineral density and bone mineral contents from childhood into young adulthood. J Clin Densitom 19(2):180-191.

5. National Osteoporosis Foundation (2010) Clinician's guide to prevention and treatment of osteoporosis. National Osteoporosis Foundation 1-56, Washington, DC, USA.

6. Standing Committee on the Scientific Evaluation of Dietary Reference Intakes (1997) Dietary reference intakes for calcium, phosphorus, magnesium, vitamin D, and fluoride. National Academies press, Washington, DC, USA.

7. Krause M, Wheeler TL, Snyder TE, Richter HE (2009) Local effects of vaginally administered estrogen therapy: A Review. J Pelvic Med Surg 15(3): 105-114

8. McCullough M (2011) New drugs for osteoporosis. Australian Prescriber 34(6): 176-181.

9. Howe TE, Shea B, Dawson LJ, Downie F, Murray A, et al. (2011) Exercise for preventing and treating osteoporosis in postmenopausal women. Cochrane Database Syst Rev 7.

10. Layne JE, Nelson ME (1999) The effects of progressive resistance training on bone density: A review. Med Sci Sports Exerc 31(1): 25-30.

11. Richardson NSM, Miller LE, Wootten DF, Ramp WK, Herbert WG (2007) Concentric and eccentric isokinetic resistance training similarly increases muscular strength, fat-free soft tissue mass and specific bone mineral measurements in young women. Osteoporosis Int 18(6): 789796.

12. Abrahin O, Rodrigues RP, Marçal AC, Alves EA, Figueiredo RC, et al. (2016) Swimming and cycling do not cause positive effects on bone mineral density: A systematic review. Revista Bras Reumatol 56(4): 345351 .

13. Cashman KD (2004) Diet and control of osteoporosis Functional foods, ageing and degenerative disease. Woodhead Publishing Limited, Cambridge, UK, pp. 83-114.

14. Levine MA (2012) Assessing bone health in children and adolescents. Indian J Endocrinol Metab 16(2): 205-212.

15. Slavin JL, Lloyd B (2012) Health benefits of fruits and vegetables. Adv Nutr 3(4): 506-516.

16. Lim LS, Hoeksema LJ, Sherin K (2009) Screening for osteoporosis in the adult US population: ACPM position statement on preventive practice. Am J Prev Med 36(4): 366-375.

17. Grainge MJ, Coupland CA, Cliffe SJ, Chilvers CE, Hosking DJ (1998) Cigarette smoking, alcohol and caffeine consumption, and bone mineral density in postmenopausal women. The Nottingham EPIC Study Group. Osteoporos Int 8(4): 355-363. 
(C) (P) This work is licensed under Creative Commons Attribution 4.0 License

To Submit Your Article Click Here: $\quad$ Submit Article

DOI: $10.32474 /$ OAJCAM.2018.01.000115

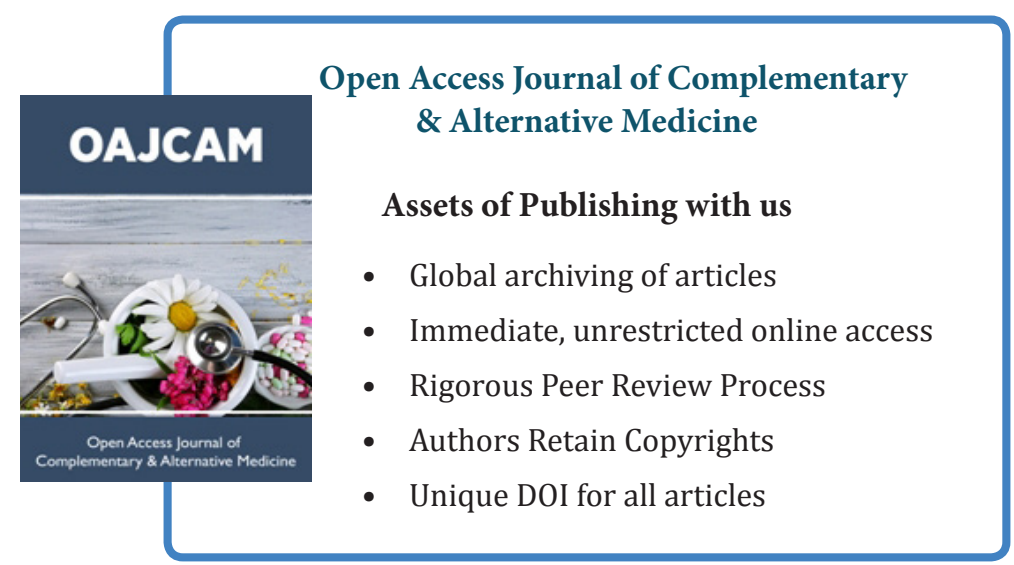

